\title{
Polymeric nanoparticles as therapeutic agents against coronavirus disease
}

\author{
Letícia Emiliano Charelli ${ }^{\circledR}$ - Gabriela Calidone de Mattos ${ }^{\circledR} \cdot$ \\ Ariane de Jesus Sousa-Batista ${ }^{\circledR} \cdot$ José Carlos Pinto $『$ • \\ Tiago Albertini Balbino ${ }^{D}$
}

Received: 18 August 2021 / Accepted: 30 December 2021 / Published online: 10 January 2022

(C) Springer Nature B.V. 2022

\begin{abstract}
Nanotechnology has the potential to improve the combat against life-threatening conditions. Considering the COVID-19 scenario, and future outbreaks, nanotechnology can play a pivotal role in several steps, ranging from disinfection protocols, manufacture of hospital clothes, to implementation of healthcare settings. Polymeric nanoparticles are colloidal particles with size ranging from 10 to $999 \mathrm{~nm}$, composed of natural or synthetic polymers. The versatility of polymeric-based nanoparticle engineering can provide (i) specificity, (ii) tunable release kinetics, and (iii) multimodal drug composition, making it possible to overcome common limitations encountered during traditional drug development. Consequently, these particles have been widely used as drug delivery systems against several diseases, such as cancer. Due to inherent competitive
\end{abstract}

Letícia Emiliano Charelli and Gabriela Calidone de Mattos contributed equally.

L. E. Charelli · A. de Jesus Sousa-Batista .

T. A. Balbino $(\varangle)$

Nanotechnology Engineering Department, Alberto Luiz

Coimbra Institute for Graduate Studies and Research

in Engineering, Federal University of Rio de Janeiro,

Rio de Janeiro, Brazil

e-mail: tiagoab@pent.coppe.ufrj.br

G. C. de Mattos · J. C. Pinto

Chemical Engineering Department, Alberto Luiz

Coimbra Institute for Graduate Studies and Research

in Engineering, Federal University of Rio de Janeiro,

Rio de Janeiro, Brazil advantages, polymeric-based nanoparticles hold astonishing potential to counteract the new coronavirus disease (COVID-19). For this reason, in the present study, the latest advancements in polymer-based nanotechnology approaches used to fight against SARS-CoV-2 are compiled and discussed. Moreover, the importance of forefront in vitro technologies - such as 3D bioprinting and organ-on-chip - to evaluate the efficacy of nanotherapeutic agents is also highlighted.

Keywords Nanomedicine $\cdot$ Nanobiotechnology SARS-CoV-2 · COVID-19 · Coronavirus · Drug delivery $\cdot$ Polymeric nanoparticles $\cdot$ Polymer

\section{Introduction}

SARS-CoV-2 is a betacoronavirus that was firstly discovered in bronchoalveolar lavage samples, obtained from patients with pneumonia in the city of Wuhan, Hubei province, China, in December 2019. SARSCoV-2 infection leads to COVID-19 disease, which is an acute respiratory infection that has high transmissibility and global distribution, promoting massive death rates with significant economic losses (Gennaro et al. 2020).

To counteract COVID-19 and future outbreaks, robust, repeatable, and cost- and time-efficient vaccines and and new drug platforms, as well as prophylactic methods, must be developed and 
validated. Considering this scenario, nanotechnological approaches have been extensively reported and encouraged worldwide as a strategy to fight and prevent SARS-CoV-2 infection (Singh et al. 2020).

Advances in nanotechnology engineering have impressively leveraged diversified areas of knowledge, such as materials, chemical, and tissue engineering as well as nanomedicine. Such advancements are strictly correlated with the nanometer scale of the materials (Chauhan et al. 2020). Compared with conventional therapeutic methods, nanostructured materials can overcome common limitations related to the specificity of the target tissue, the release rate, and biodegradation of bioactive molecules as well as act as carries of hydrophobic and hydrophilic compounds with fewer side-effects (Sahandi Zangabad et al. 2017). Moreover, sophisticated strategies to enhance the therapeutic potential of nanomedicines such as functionalization, passivation, and loading of multiple drugs in a single carrier can enable steadier biological responses compared with traditional methods (Zhou et al. 2020).

Due to their inherent colloidal stability and surface activity, nanomaterials can also escape undesirable interactions with the immune system and extend their circulation time in the bloodstream (Zhou et al. 2020). Otherwise, they can also be engineered to facilitate the interaction with the immune system, turning these materials into interesting tools to act as adjuvants and virus-like particles in vaccines (Zhou et al. 2020).

To date, many nano-designed systems have been clinically approved for human use by the Food and Drug Administration (FDA), including liposomal, nanocrystal, inorganic, protein, and polymeric materials (Anselmo and Mitragotri 2019). Particularly, polymer-based nanoparticles have been broadly used in nanomedicine to treat different diseases such as cancer, bone regeneration, and autoimmune diseases (Yetisgin et al. 2020). Polymeric nanoparticles can be composed of natural, synthetic, or pseudosynthetic single polymer chains or larger chain aggregates (Yetisgin et al. 2020). Moreover, they are extremely versatile regarding their conjugation, leading to enhanced biocompatibility and good control of in vivo circulation time, such properties made them one of the top FDA-approved nanodrugs (Anselmo and Mitragotri 2019).
The high surface area of the polymeric NPs allows a highly efficient drug carrier, and the size becomes a determining characteristic at the moment of interaction with the cell membrane and in the penetration of physiological barriers (Kumari et al. 2010; Milane and Amiji 2021). In addition, these nanosystems have unique physical-chemical characteristics such as (i) prolonged blood circulation time; (ii) reduced adverse effects; (iii) ability to protect therapeutic agents from degradation, increasing the stability, bioavailability, and pharmacokinetics of drugs; (iv) ease of chemical modification; (v) controlled drug release; and (vi) improved therapeutic effects (Gao et al. 2020; Yang 2021; Sun et al. 2021). Due to its advantages, nanostructured polymers have been considered as tools to fight against COVID-19 (Zhang et al. 2020).

Herein, we discuss the latest advancements regarding the use of polymer-based nanotechnology approaches to fight against SARS-CoV-2. Moreover, the importance of developing a biologically relevant platform for in vitro evaluation of polymeric vaccines/nanosystems is also highlighted.

\section{Structure and pathophysiology of SARS-Cov-2}

Viral infections cause morbidity and mortality worldwide, leading to significant economic losses (Yetisgin et al. 2020). The latest reported viral infections include the severe acute respiratory syndrome (SARS), the Middle East respiratory syndrome (MERS), the Ebola disease, the Zika virus influenza, and the chikungunya disease (Parvez and Parveen 2017).

Coronaviruses constitute a large family of viruses that can induce diseases ranging from a simple cold to SARS. The cluster of coronavirus SARS-CoV-2 was originally identified in Wuhan, China, in December 2019, leading to the COVID-19 disease outbreak (Gennaro et al. 2020). There are four genera of coronavirus: alpha, beta, gamma, and delta. SARS-CoV-2 is a betacoronavirus ranging from 60 to $140 \mathrm{~nm}$ in diameter. Its morphology consists of a singlestranded positive-sense RNA genome with a glycoprotein spike transmembrane within the capsid, ranging from 9 to $12 \mathrm{~nm}$ (Mousavizadeh and Ghasemi 2020) (Fig. 1).

The pathogenesis mechanism of SARS-CoV-2 is intrinsically related to its spike protein (Zhang et al. 
(a)
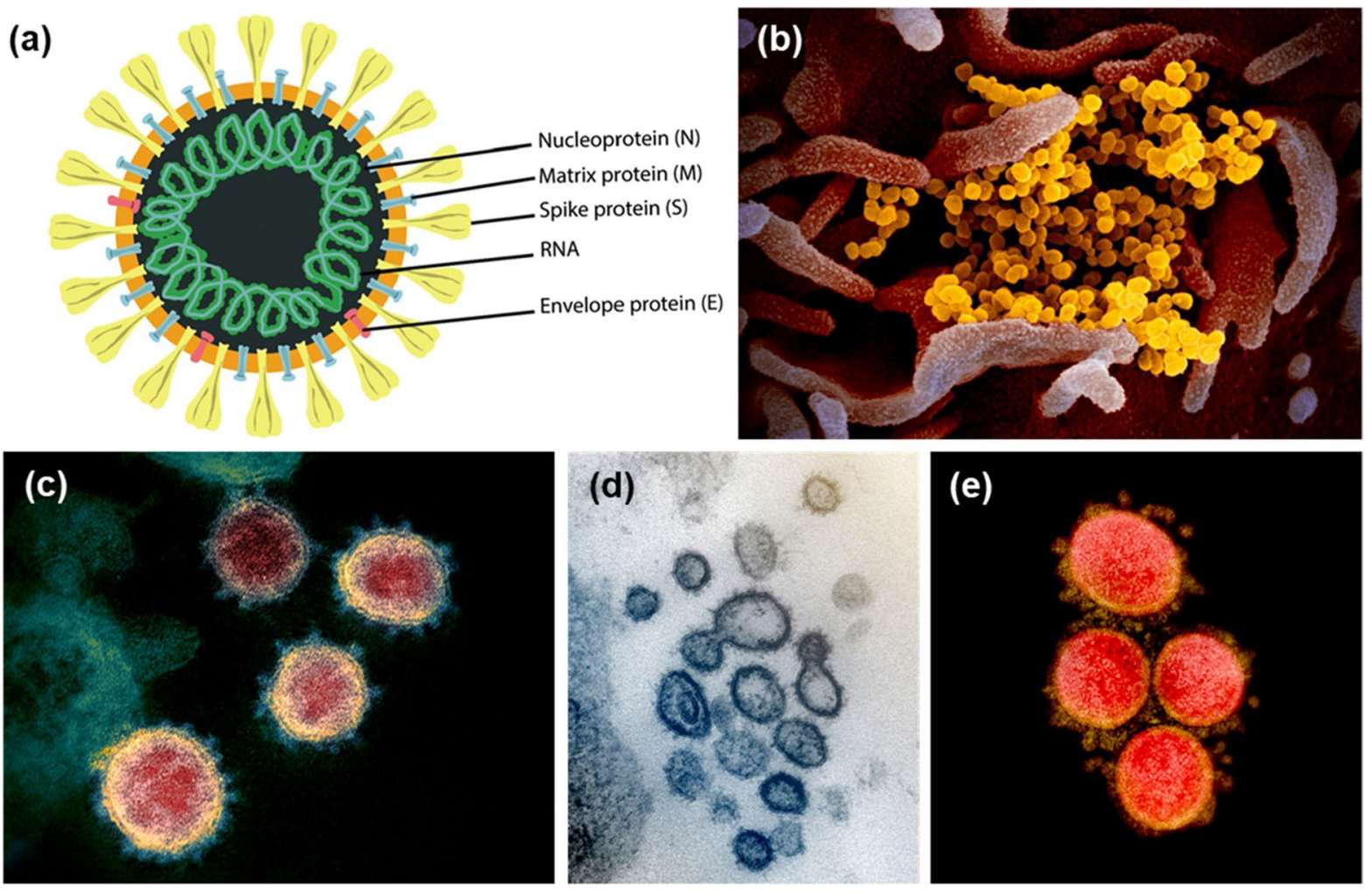

Fig. 1 a Schematic representation of SARS-CoV-2 [Udugama et al. 2020Medhi et al. 2020 adapted from Udugama et al., 2020 with permission from ACS Nano](2020). b Scanning electron microscopy of SARS-CoV-2 (yellow) isolated from a patient in the USA, emerging from the surface of cells (pink).
Credit: NIAID-RML. c-e Transmission electron microscopy of SARS-CoV-2 isolated from a patient in the USA. The crownlike spikes on the external edge of SARS-CoV-2 give coronaviruses their name. Credit: NIAID-RML (2020)
2020). The infection mechanism into the host cells is driven by five important steps: (i) binding with the host receptor (angiotensin-converting enzyme 2, ACE2); (ii) direct membrane fusion or endocytosis between the virus and the plasma membrane; (iii) biosynthesis of viral RNA; (iv) maturation; and (v) release (Mousavizadeh and Ghasemi 2020). Clinically, early-stage infected patients may experience fever, dry cough, fatigue, and difficulty to breath.

SARS-CoV-2 is the most contagious in its class, affecting mainly the lower respiratory system, leading to viral pneumonia and progressive respiratory failure (Gennaro et al. 2020). Moreover, its systemic pathogenicity can also affect organs such as the heart, liver, and kidney and the central nervous system. Its transmission can occur directly (human-tohuman) or indirectly (airborne and infected objects) (Mousavizadeh and Ghasemi 2020).
To date, it is estimated that over 3,287,082 million deaths have been caused by SARS-CoV-2 (Organization 2021). Treatments using antiviral drugs (including interferon, ribavirin, arbidol, and remdesivir), antiviral antibodies, and anti-inflammatory (such as colchicine) have been reported with certain levels of positive results (Pooladanda et al. 2020). Moreover, the elucidation of the SARSCoV-2 genome has allowed the development of several vaccine candidates based on its pathogenesis mechanism (Forni and Mantovani 2021). The strategy for the construction of the SARS-CoV-2 vaccines includes the use of an attenuated virus, inactivated virus, SARS-CoV-2 proteins (such as the spike protein), naked DNA, mRNA, and other viral vectors (Forni and Mantovani 2021). A detailed review regarding the vaccines used to combat COVID-19, their features, and challenges that still 
must be faced was discussed elsewhere (Forni and Mantovani 2021).

However, the constant genetic recombination of SARS-CoV-2 often leads to high rates of mutation (Islam et al. 2020). To date, many variants of COVID19 have been detected around the world, including Brazil, South Africa, and the UK variants (Fontanet et al. 2021). Consequently, novel therapeutic strategies are still required to prevent and treat SARSCov-2 infections, including the possible variants and reinfection in patients. In this scenario, nanotechnology can provide a versatile and powerful platform to combat COVID-19 infection and future outbreaks.

\section{Does size matter? Nanomedicine as a weapon against SARS-CoV-2}

According to the strict definition, structures with dimensions between 1 and $100 \mathrm{~nm}$ in size in at least one dimension are conceptually called nanostructures (Manchanda et al. 2019). The properties of nanostructured materials are different from the bulk due to their high surface area/volume ratio and the quantum effects from the nanoscale (Manchanda et al. 2019). The combination between nanotechnology and biotechnology (i.e., nanobiotechnology) is an emerging field with applicability ranging from drug development, disease modeling, diagnosis, bionanosensors, tissue engineering, and medical imaging (Manchanda et al. 2019; Bandopadhyay et al. 2019).

Nanoparticle-based drug delivery systems can be defined as nanocarries designed to deliver drugs, vaccines, and therapeutic compounds (Wang et al. 2021b). Due to their size, these nanostructured delivery systems can penetrate biological barriers and deliver the drug in the tissue of difficult access, such as the central nervous system. Moreover, they can also evade the immune system, e.g., phagocytes, prolonging their time in the bloodstream (Wang et al. 2021b). The possibility of loading complex drugs into nanocarriers increasing cellular uptake, half-life, bioavailability, and consequently, water-solubility makes these nanostructured systems the target of numerous scientific studies at the present moment (Bandopadhyay et al. 2019; Manek and Petroianu 2020).

Nanostructured polymeric drug delivery systems can be designed as nanosphere, nanocapsule, or as conjugate, and the loaded drugs can be entrapped, encapsulated, or even bounded (Patra et al. 2018). The most reported nanocarriers active delivery systems available in the last two decades are lipid-based nanoparticles, polymeric nanoparticles, dendrimers, nanoemulsions, nanosuspensions, metallic nanoparticles, nanomicelles, carbonaceous carriers, and milk-derived carriers (Bandopadhyay et al. 2019; Patra et al. 2018). All the systems aforementioned have their particular advantages and limitations, and several of them are already approved by regulatory authorities that use determined criteria for marketing authorization for products that have biomedical applications as the final proposal (Bandopadhyay et al. 2019; Montanheiro et al. 2020).

The improvement of nanoparticle research has also been focused on early diagnosis in addition to carrying actives for the treatment of diseases such as cancer, diabetes, and neurodegenerative and cardiovascular diseases (Bandopadhyay et al. 2019). Additionally to drug delivery and diagnosis, nanotechnology interventions can also play a pivotal role in the prevention of COVID-19 as antiviral coaching of clothes, masks, face shields, and equipment (Weiss et al. 2020) (Fig. 2A-B). For these reasons, studies aimed at improving existing systems and new systems under development are increasingly focused on employing nanotechnology.

In the next sections, we discuss the specific contributions that polymeric nanoparticles can offer to counter SARS-CoV-2 pandemic and future outbreaks.

\section{Polymeric nanoparticle-based approaches to boost COVID-19 therapeutics}

Manufacturing: synthesis and functionalization

Considering all the potential of using polymericbased nanoparticles to treat several human conditions, in this section, we highlight the advantages of using polymeric nanoparticles as a strategy to fight the SARS-CoV-2 infection and disease.

Two extremely advantageous polymers, since they have already been approved by the FDA, widely studied and with a well-established synthesis route are the PLA and PLGA. The most frequently used approaches in the manufacturing of PLA or PLGAbased drug delivery nano-sized systems are the nanoprecipitation and emulsification processes as shown 


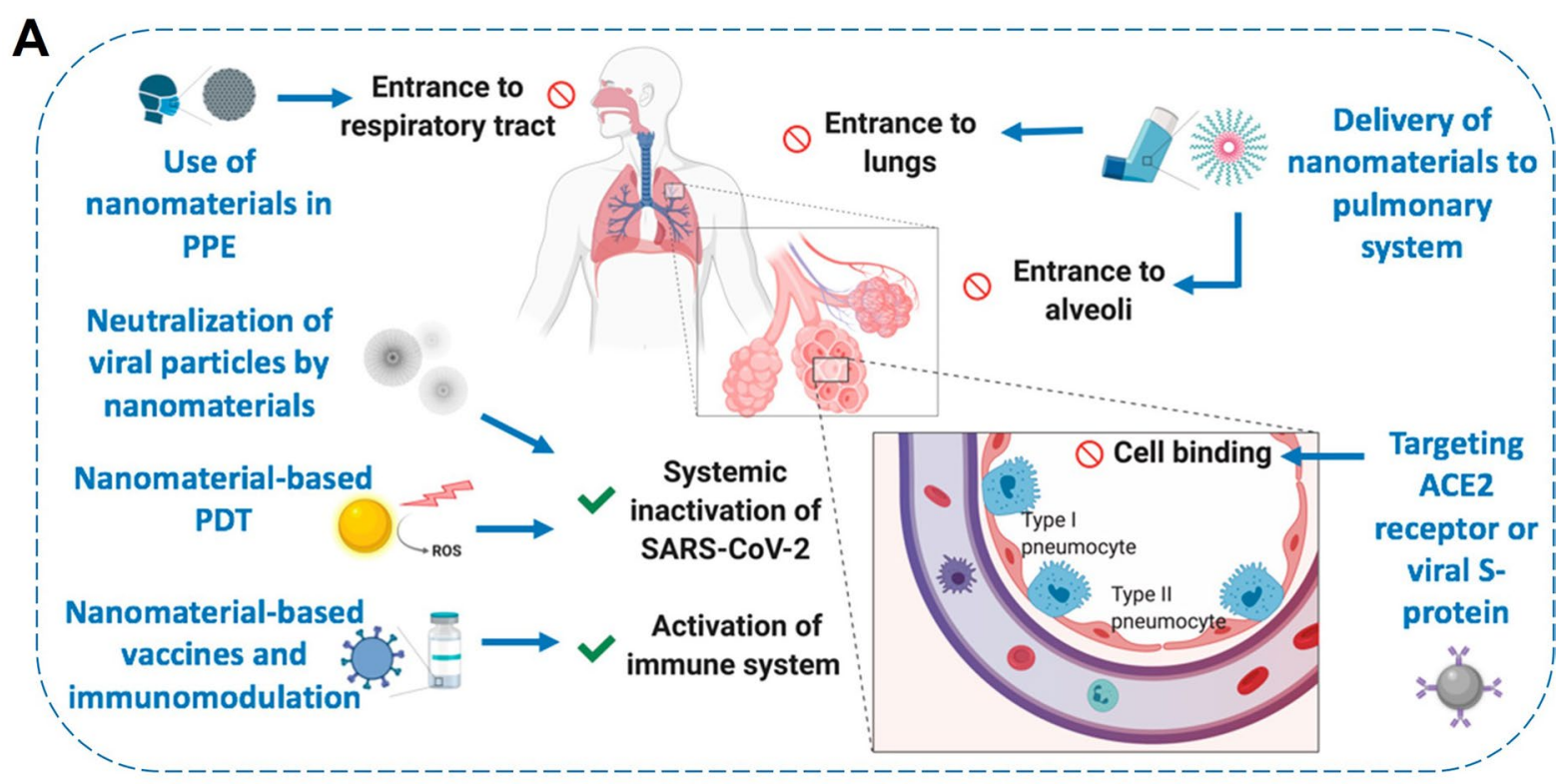

B

\section{CORONAVIRUS \& SARS-COV \& NANO}

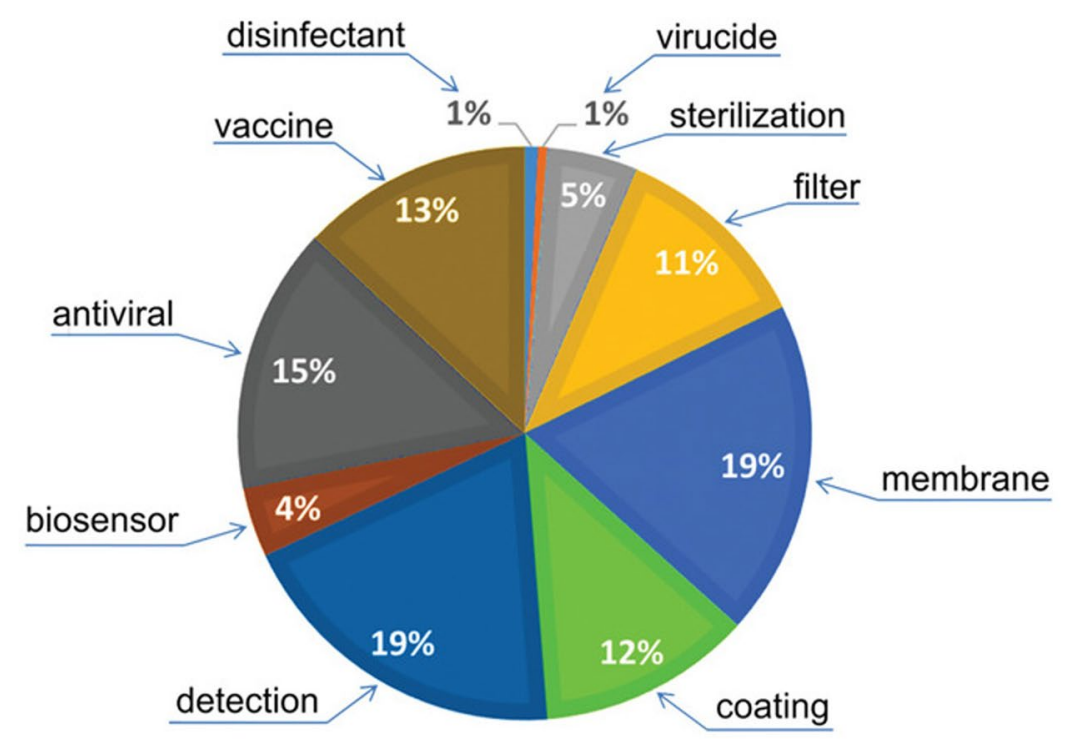

Fig. 2 A The versatility of nanotechnology against SARS$\mathrm{CoV}-2$. Personal protective equipment (PPE). Angiotensinconverting enzyme 2 (ACE2). Photodynamic therapy (PDT). [Weiss et al. 2020Ruiz-Hitzky et al. 2020 Adapted from Weiss

in Fig. 3A (Bandopadhyay et al. 2019; Rezvantalab et al. 2018). The synthesis processes of polymeric nanoparticles play a fundamental role in the final performance of the system. Therefore, properties such as shape, size and size distribution, surface charge, and et al., 2020, with permission from ASC Nano](2020). B Patent distribution regarding "coronavirus and nano." Data acquired from SPACENET [Adpated from Hitzky et al., 2020 with permission from ASC Nano](2020)

stability must be controlled for an efficient application as well as to define the administration route (Rezvantalab et al. 2018).

Nanoprecipitation processes normally begin with a solution that contains the previously prepared 
A
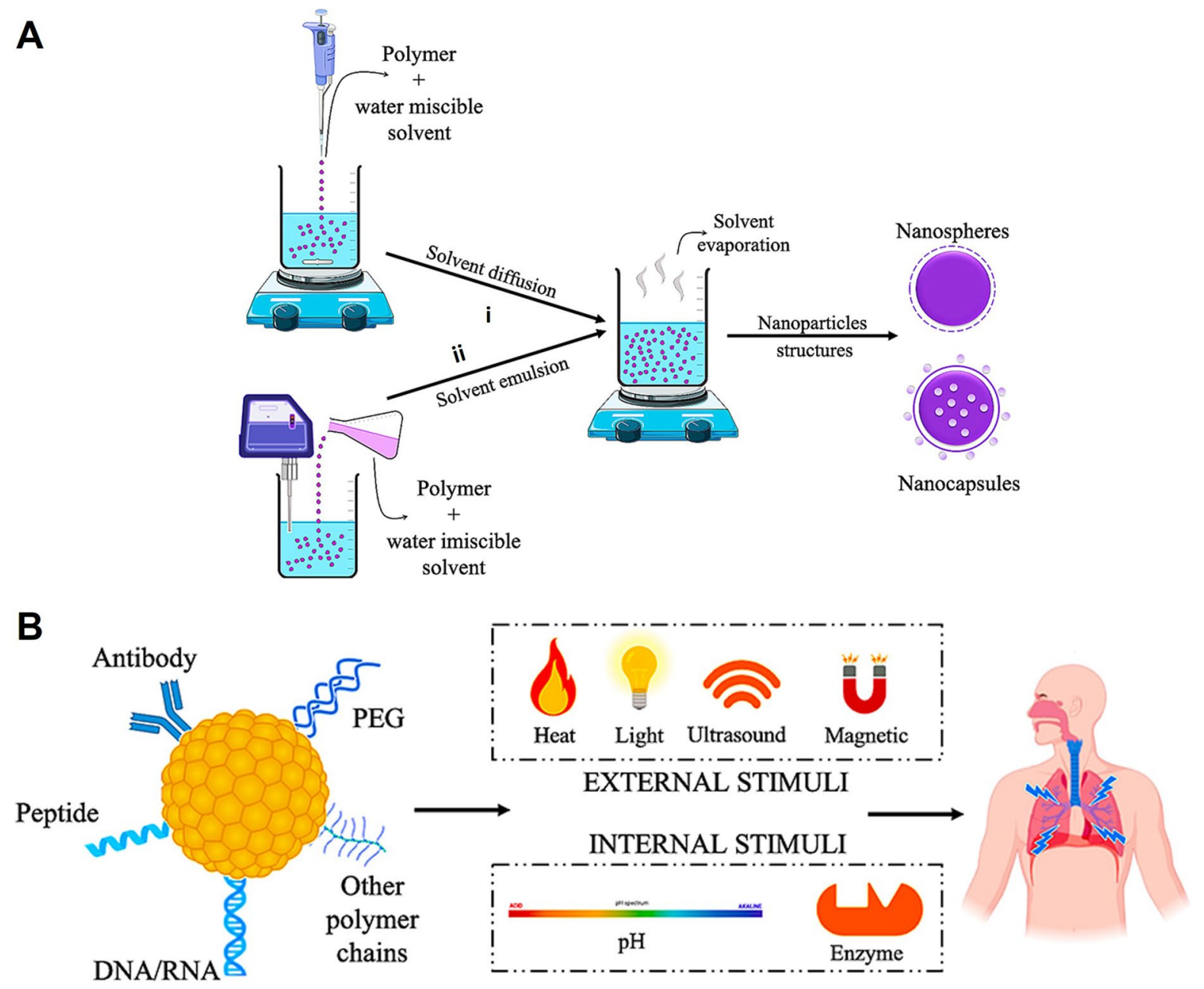

Fig. 3 A Methods of the preparation of polymeric nanoparticles: Nanoprecipitation (i) and emulsification (ii) processes. The first process requires the controlled addition of the polymer solution and hydrophilic solvent in water. The second process requires the emulsification of an insoluble dispersed phase. In both processes, it is necessary to remove the sol-

polymer material and the drug, while a process variable (temperature, addition of non-solvent or salts, modification of the $\mathrm{pH}$ value) is manipulated to reduce the solubility of the solutes in the analyzed solvent and force the precipitation (Fig. 3A). Nanoprecipitation processes often do not require the addition of surfactants, although the purification of reagents plays an important role to avoid the formation of agglomerates and allow the control of particle nucleation and growth (Jain et al. 2018). vent in order to obtain the final nanoparticles. B Nanoparticle functionalization strategies for targeted delivery. When the nanoparticles are superficially functionalized, they are able to respond to external or internal stimuli, including variations of heat, the incidence of light or waves, the presence of the magnetic field, $\mathrm{pH}$, or recognition of specific receptors

Nevertheless, nanoprecipitation processes can be affected by the very same variables described before, being also subject to a large number of process parameters that must be defined and controlled (Jain et al. 2018), demanding once more time-consuming process optimization. Consequently, the process productivity is also low, given the usually low concentrations of polymer in the solution. Nanoprecipitation processes can also lead to very expensive operations, given the necessity to recover, purify, and recycle the 
solvent and be environmentally incorrect. Finally, the encapsulation efficiencies can be minimal, as the drug is partitioned between the continuous solvent phase and the nanoprecipitated polymer phase.

The emulsification of previously prepared polymer solutions and the subsequent solvent evaporation have been frequently applied to prepare nanocarriers loaded with both hydrophilic and lipophilic drugs (Fig. 3A) (Saraogi et al. 2010; Gorle and Gattani 2009). In this case, the physicochemical characteristics of the obtained nanospheres can be affected by (i) the solubility of the active compound in the solvent; (ii) the nature of the organic solvent; (iii) the concentration of polymer in the considered organic solvent; (iv) the nature and concentration of the surfactant used to keep the dispersion stable; and (v) the stirring speed. The large number of process parameters that must be defined and controlled can be regarded as a negative point of solvent evaporation processes (Jain et al. 2018; Kulterer et al. 2011), demanding time-consuming process optimization. Moreover, the process productivity is usually low, due to the usually low concentrations of polymer in the solution and the low fractions of the dispersed phase. This can lead to high-cost operations, given the necessity to recover, purify, and recycle the solvent. Besides, this process can be environmentally incorrect, due to the large amounts of heat required to evaporate and purify the solvent and the large amounts of surfactants required to stabilize the system. PLGA nanoparticles loaded with different bioactive compounds have been widely produced through combined emulsification and solvent evaporation (Bandopadhyay et al. 2019).

Regarding the drug, many possibilities are being studied and already have potential results. Among them are remdesivir, favipiravir, lopinavir/ritonavir, chloroquine, daclatasvir, and atazanavir (Witika et al. 2020; Aleissa et al. 2021; Eslami et al. 2020; Rahmani et al. 2020). The incorporation of these drugs in nanodimensioned systems can improve the solubility and biodistribution and increase the half-life of the active when compared to the assets in their free form or in microsystems (Sur et al. 2019). Chloroquine may be interesting because it can inhibit the endocytosis of nanoparticles, which is what happens in the viral infection of SARS-CoV-2; however, there are still no studies that are considered effective for this virus (Medhi et al. 2020). In October 2020, the FDA approved the use of the antiviral drug Veklury (remdesivir) for the hospital treatment of COVID-19 (FDA approves first treatment for COVID-19. 2020). The drug in question was selected for study because it is already used to treat another virus (Ebola) acting as an RNA-dependent RNA polymerase (RdRP) inhibitor and because it has a positive efficacy against the COVID-19 virus (Witika et al. 2020). Thus, it can be suggested that remdesivir has advantages over others in the process of testing by encapsulating in polymeric nanoparticles.

The functionalization (i.e., vectorization, immobilization, or bioconjugation) of nanoparticle surfaces with targeting ligands has been frequently performed to deliver drugs or genes directly to the target sites, minimizing the spread of the compound in other tissues (Manek and Petroianu 2020). Surface modification is performed to increase the affinity between the delivery system and the diseased tissue (Bandopadhyay et al. 2019; Jazayeri et al. 2016). Therefore, the selective targeting of the nanocarrier depends on the capacity of the anchored ligand to recognize the receptor expressed at the target site and vice versa (Bandopadhyay et al. 2019). Antibodies, peptides, proteins, aptamers, and small molecules are examples of biomolecules that have been used for targeted delivery, as shown in Fig. 5. The functionalization of the nanoparticles can be conducted after the preparation of the nanocarriers or through the insertion of the desired chemical functions during polymerization and preparation of the polymer nanoparticles (in situ functionalization) (Fig. 3B) (Pinto et al. 2020).

Two fundamental approaches are used for targeting delivery: passive and active targeting (Attia et al. 2019). Passive targeting does not depend on a ligand for specific binding, being directly related to the characteristics of the nanoparticulate carrier (including size and circulation time) and of the tissue characteristics (including vascularity and leakiness) (Bandopadhyay et al. 2019). For example, tumors typically present differentiated biological conditions, such as inflammation and hypoxia, making the endothelium of blood vessels more permeable to larger particles than usually observed in healthy tissues (Bandopadhyay et al. 2019). Active targeting can be achieved by inserting specific ligands on the surfaces of the nanocarrier systems (Attia et al. 2019). With this outer decoration, the nanocarrier can bind to the receptors present on the surface of the targeted site (Bandopadhyay et al. 2019), allowing the increase of active 
delivery to the specific target when compared to the conventional use of free drugs or with passive targeting methods.

Functionalization can also be used to avoid the uptake of nanocarriers by cells that do not constitute the target. For example, the reticuloendothelial system (RES) is composed of cells that have reticular and endothelial characteristics (Kalyane et al. 2018). These cells are endowed with phagocytic capacity, performing the defense against generalized infections, among many functions (Kalyane et al. 2018). To prevent the RES from uptaking the nanoparticles, their surface can be modified with hydrophilic groups or can be conjugated with polyethylene oxide containing amphiphilic polymers (Manek and Petroianu 2020). Escaping from the RES clearance, the nanoparticles are not captured by the mononuclear phagocyte system (MPS), remaining in the organism until action is complete (Bandopadhyay et al. 2019). A possible disadvantage of nanoparticles used for active delivery is related to capture by the RES, clearing particles from the circulatory system and driving them to the liver or the spleen. To solve this problem and avoid recognition by the RES, functionalization can be performed through the addition of polyethylene glycol (PEG) to the surface, also known as PEGylation (Bandopadhyay et al. 2019). This strategy was discovered and has been used in medicinal systems for years. In 1994, for example, Gref et al. observed an increase in the circulation time of PLGA polymeric nanoparticles with a reduction in uptake by the liver when compared to the non-pegylated nanoparticles (Gref et al. 1994). Considering viral infection, Chan et al. (2017) studied the presence of two linear PEGs on interferon $\alpha 2$ and antiviral/antiproliferative protein used to treat hepatitis $\mathrm{C}$ infection. This strategy also resulted in longer plasma circulation times than conventional interferon (Chan et al. 2017).

PEG is a non-immunogenic polymer widely used for therapeutic purposes because of its non-toxic properties and the possibility to enhance drug solubility and particle stability (Veronese and Mero 2008). PEGylation can lead to an increase in the circulation time of nanoparticles in the blood, providing more time for transport mechanisms to occur and improving the therapeutic efficiency (Manek and Petroianu 2020). Similarly, the use of poly(vinyl acetate) (PVA) in humans has been approved by the FDA, given its low toxicity and good biocompatibility properties, so that PVA has been widely applied for the stabilization of nanoparticulate polymer systems (Manek and Petroianu 2020).

Some functionalized nanosystems have already been tested in clinical trials, showing effective affinity results, strong bindings, and simple chemistry as advantages and high production costs, low circulation times, and potential immunogenicity as factors that still need to be improved (Attia et al. 2019). However, for most systems under study, the ideal surface density and the disposition of the conjugating agents are not yet well-established. It has not yet been defined how binders can affect the properties of nanocarriers, such as charge, stability, toxicity, and immunogenicity. Thus, in order to accurately predict the impact of surface modification on the therapeutic performance of nanoparticulate polymeric carriers, additional research is still needed.

As mentioned before, the virus replication process takes place inside the human cell after entry through protein receptors on the cell surface. Considering the functionalization strategy that can enhance the treatment against SARS-CoV-2, a suggested alternative is the neutralization of the viral particle after the virus enters the respiratory tract but before the virus enters the cell, inhibiting replication. As the coronavirus is a natural nanoparticle that interacts - precisely and efficiently - with the organism, one possibility of therapy is to create an artificial nanoparticle capable of interacting with the host's sites before the virus. Therefore, nanoparticles functionalized with ACE2 and CD147 proteins could saturate the receptors present in cells, reducing their availability and blocking viral entry in our cell (Jagaran and Singh 2020). Another interesting functionalization strategy that could be applied in addition to the suggested above is PEGylation. Thus, the recognition and capture of the nanoparticle by macrophages could become more difficult, increasing the circulation time (Bandopadhyay et al. 2019; Manek and Petroianu 2020).

Promising polymeric nanoparticles for COVID-19 disease and sequelae

Vaccines using nanoscale systems are expected to increase the immunogenicity of the vaccine antigen by controlling delivery to cells. Furthermore, they can support the simultaneous delivery of an antigen with an immunomodulator (Kim et al. 2014). For 
respiratory viral diseases in general, there are already studies of nanoparticulate vaccines administered intranasally that show promising results (Al-Halifa et al. 2019). The nanoparticulate PLGA (polylacticco-glycolic acid) is already used in the delivery of hepatitis B virus antigens, tetanus toxoid, and Mycobacterium tuberculosis antigen, for example (Bhardwaj et al. 2020). PLGA NPs, chitosan, polyanhydride, poly- $\gamma$-glutamic acid, and $\mathrm{N}$-(2-hydroxypropyl) methacrylamide/N-isopropylacrylamide (HPMA/ NIPAM) with sizes varying between 12 and $800 \mathrm{~nm}$ are being developed and improved to be applied in cases of the influenza virus (H1N1), bovine parainfluenza 3 virus (BPI3V), swine influenza virus (H1N2), and respiratory syncytial virus (RSV), for example (Al-Halifa et al. 2019).

Considering therapies for pulmonary diseases, it is possible to describe studies that have been performed for more than 10 years and suggest that treatments based on nanosystems can be advantageous and able to overcome limitations of other conventional systems. For instance, Matsuo et al. (2009) studied the encapsulation of betamethasone by PLA homopolymers for the treatment of asthma (Matsuo et al. 2009). Saraogi et al. (2010) used gelatin nanoparticles to encapsulate rifampicin for use in tuberculosis therapies (Saraogi et al. 2010; Silva et al. 2013). Since then, nanocarriers have also been used to prepare commercial products. For example, Abraxane ${ }^{\circledR}$ is based on albumin nanoparticles loading paclitaxel for the treatment of certain types of lung and breast cancers (Jain et al. 2018).

After the onset of the COVID-19 pandemic, induced by severe acute respiratory syndrome coronavirus 2 (SARS-CoV-2), several researchers around the world modified the conventional objectives of their research and engaged in the search for alternatives that could minimize the effects of the virus.

In the context of mRNA-loaded vaccines for COVID-19, the ones already available were developed by Pfizer/BioNTech and Moderna, for example, and are composed of cationic lipid nanoparticles. mRNA vaccines are more advantageous over DNA or whole virus vaccines because the mRNA is noninfectious, with a short and immunogenic half-life, and can be transported safely and efficiently in vivo (Nanomedicine and the COVID-19 vaccines 2020). Similarly, to lipid-based devices, polymer systems constitute feasible alternatives for the delivery of genetic material and other immunomodulatory compounds for vaccination. Natural and synthetic polymers, such as chitosan and polypeptides, respectively, are able to form nanostructured vaccine platforms, where cationically charged groups from polymer chains can electrostatically bind to the negatively charged mRNA, DNA, or nonreplicating adenovirus vector-based (Kim et al. 2014).

Thus, nanostructured polymer systems can be manipulated with regard to molecular weight and encapsulation conditions, enabling the controlled release of pharmaceuticals and simultaneously constituting robust alternatives to induce immune responses for the treatment of viral infections (Kim et al. 2014; Zhang et al. 2017). Polymeric NPs have the potential to inhibit the effects of viral infections due to their possibility of encapsulation of antiviral agents improving bioavailability control release and reducing side effects (Abo-zeid et al. 2020).

Among some of the advantages presented by polymeric NPs for use against the SARS-CoV-2 virus are the high stability of the systems, control of drug delivery, biocompatibility, manipulation and adequacy of physical and chemical properties, compatibility with hydrophilic and hydrophobic drugs, and also the preparation through simple and suitable methods (polymer, NP size, desired administration route, and the drug carried, among others) (Yang 2021; Bhardwaj et al. 2020).

In this context, polymeric nanoparticles have become relevant systems in this current pandemic situation since they can be adjusted according to the specific application and can be used mainly in (i) diagnostic sensors for detections of viral pathogens, such as protein S, for example (Yang 2021; Drobysh et al. 2021); (ii) textiles in facial masks acting as barriers to the virus (Militky et al. 2021); and (iii) controlled release systems for treatment or prevention, such as vaccines (Yang 2021). Considering the group of treatments and vaccines, historically the polymeric NPs have already been exploited for other viral and infectious diseases because they allow administration in a systemic way or through inhalation or intranasal route reaching the lungs directly (Rana 2021).

A literature search was done using the terms ((COVID OR SARS) AND Nanoparticle* AND Poly$\left.m e r^{*}\right)$ by using the Web of Science (WoS) database in May 2021 and found only 42 research articles that were published. However, 35 have been published 
from 2019 to date, and many of these results were not appropriate to the topic of this review. Among the researches that relate polymeric nanoparticles aimed at applications in the COVID-19 virus, solutions that seek to reduce or prevent the entry of the virus into the cell for replication, diagnosis and detection of the virus, and treatment after infection stand out.

In the search, articles were found that aim of treatment as (i) to study of how hybrid nanoparticles (lipid and polymeric) could serve as vehicles for improving transfection of messenger RNA (mRNA) (Siewert et al. 2020); (ii) the comparison of the uptake of poly(glycerol-adipate) nanoparticles fluorescently labeled with rhodamine B isothiocyanate in different rhinovirus infected cells (Abo-zeid et al. 2020); and (iii) the study of a systematic computational investigation on lisinopril-capped remdesivir-loaded PLGA nanoparticles. A binding model confirmed two interactions, the first was between lisinopril and ACE receptor of the cells, and the second was between remdesivir and its intracellular targeting protein $(\mathrm{Wu}$ et al. 2020).

Once the virus (carrier of the spike protein $(\mathrm{S})$ ) is in the host's body, it needs to find the ACE2 sites present on cell surfaces in order to enter the cell and replicate. As a way to prevent the onset of viral infection or prevent further replication from occurring, Neufurth et al. (2020), Neufurth et al. (2021), and $\mathrm{Li}$ et al. (2021) studied ways to avoid the encounter between virus S protein and the ACE2 receptor (Neufurth et al. 2020; Neufurth et al. 2021; Li et al. 2021). It was found that nanoparticles of the magnesium salt of inorganic polyphosphate (polyP) is capable of binding to the protein S (Neufurth et al. 2020), and from that, dexamethasone/quercetin was loaded to study the system effects on the respiratory epithelium. As conclusions, the authors identified that the drugs caged into the polyP showed better results of gene expression compared to the individual drugs alone and caused an increased expression of mucin (Neufurth et al. 2021). Additionally, Li et al. (2021) developed multifunctional alveolar macrophage (AM)-like nanoparticles (NPs) with photothermal inactivation against the virus (Li et al. 2021). In the system, AM membranes with coronavirus receptors internalize (coating) PLGA nanoparticles doped with a photothermal molecule. In this way, the virus binds to the nanoparticles before it finds the cell membrane and is then photothermally deactivated (Li et al. 2021).
For detection and diagnostic systems, PLGA nanovesicles loaded with 3,3',5,5'-tetramethylbenzidine (TMB) chromogen were developed for the detection of various respiratory-infecting viruses (Khoris et al. 2021), and nanoparticles of vinyl-modified AIE-active TPE-1VBC, styrene (St), divinylbenzene (DVB), and maleic anhydride (MAH) as comonomers via self-stable precipitation polymerization were studied to be used in COVID-19 diagnostic tests (Wang et al. 2021a). It is noteworthy that among the 8 works that correlate the selected keywords, 3 use PLGA as the polymer matrix of the developed system (Table 1).

\section{D bioprinting and organ-on-chip as frontier in vitro technologies for COVID-19 and future outbreaks assessment}

The use of polymer-based nanoparticles to treat infectious diseases constitutes a feasible reality. Thus, technologies that accurately predict the therapeutic performance and biological safety of nanoparticles are mandatory. In this context, 3D bioprinting and organ-on-chip devices have been reported as promisor tools toward this goal (Trampe et al. 2018; Skardal et al. 2016). These platforms have been extensively discussed as predictive in vitro models to study diseases and to evaluate nanopharmaceuticals, constituting excellent approaches to study the different reactions that COVID-19 promotes in the human body as well as the efficacy of polymer-based delivery systems.

Bioprinting and organ-on-chip combine additive manufacturing, microfabrication, microfluidics, and tissue engineering fields in order to emulate key features of living organisms (Zhang et al. 2018). Due to their advantages, both technologies have been considered powerful tools in several areas, such as drug development, disease modeling, nanobiotechnology, and tissue engineering (Park et al. 2018).

Bioprinting technology allows the biofabrication of physiologically relevant tissue models. It is possible, for example, to use patients' own cells to generate tissue-like constructs and to test specific drugs candidates. Considering the COVID-19 scenario, it would be possible to biofabricate lung models and to infect them with SARS-CoV-2 to augment the understand regarding its pathogenicity (Grigoryan et al. 2019). 
Table 1 The use of PLGA as polymeric matrix

\begin{tabular}{|c|c|c|c|c|c|c|}
\hline & Polymer & Synthesis & Functionalization & Encapsulation & $\begin{array}{l}\text { In vitro/in vivo } \\
\text { tests }\end{array}$ & Ref \\
\hline Treatment & Protamine & NI & NI & mRNA & In vitro/in vivo & $\begin{array}{l}\text { Siewert et al. } \\
\text { 2020) }\end{array}$ \\
\hline Treatment & $\begin{array}{l}\text { Poly(glycerol- } \\
\text { adipate) }\end{array}$ & NI & $\begin{array}{l}\text { Rhodamine B } \\
\text { isothiocyanate }\end{array}$ & NI & NI & $\begin{array}{l}\text { Abo-zeid et al. } \\
\text { 2020) }\end{array}$ \\
\hline $\begin{array}{l}\text { Prevention/treat- } \\
\text { ment }\end{array}$ & Silica/polyP & $\begin{array}{l}\text { "Stöber synthe- } \\
\text { sis" }\end{array}$ & NI & NI & In vitro & $\begin{array}{l}\text { Neufurth et al. } \\
\text { 2020) }\end{array}$ \\
\hline Treatment & PLGA & NI & Lisinopril & $\begin{array}{l}\text { Remdesivir/ } \\
\text { lisinopril }\end{array}$ & NI & Wu et al. 2020) \\
\hline Detection & PLGA & Double emulsion & NI & TMB chromogen & NI & $\begin{array}{l}\text { Khoris et al. } \\
\text { 2021) }\end{array}$ \\
\hline $\begin{array}{l}\text { Prevention/treat- } \\
\text { ment }\end{array}$ & PolyP & $\begin{array}{l}\text { Nanoprecipita- } \\
\text { tion }\end{array}$ & NI & $\begin{array}{l}\text { Dexamethasone/ } \\
\text { quercetin }\end{array}$ & In vitro & $\begin{array}{l}\text { Neufurth et al. } \\
\text { 2021) }\end{array}$ \\
\hline Diagnostic & $\begin{array}{l}\text { Vinyl-modified } \\
\text { AIE-active } \\
\text { TPE-1VBC, } \\
\text { styrene (St), } \\
\text { divinylbenzene } \\
\text { (DVB), and } \\
\text { maleic anhy- } \\
\text { dride (MAH) }\end{array}$ & $\begin{array}{l}\text { Self-stable } \\
\text { precipitation } \\
\text { polymerization }\end{array}$ & NI & NI & NI & $\begin{array}{l}\text { Wang et al. } \\
\text { 2021a) }\end{array}$ \\
\hline $\begin{array}{l}\text { Prevention/treat- } \\
\text { ment }\end{array}$ & PLGA & $\begin{array}{l}\text { Nanoprecipita- } \\
\text { tion }\end{array}$ & $\begin{array}{l}\text { AM cell mem- } \\
\text { branes and } \\
\text { photothermal } \\
\text { molecule }\end{array}$ & NI & In vivo & Li et al. 2021) \\
\hline
\end{tabular}

*NI, not informed

Likewise, it is also possible to replicate tissues known to have augmented expression of AC2 receptors, e.g., heart, and evaluate the efficacy of nanodrugs for that specific organ. Indeed, several studies are using 3D bioprinting technology for risk assessment of nanomaterials (Trampe et al. 2018), drug development (Peng et al. 2017), and disease modeling (Ma et al. 2018a).

Microfluidics is an area where fluids are manipulated on a micro-scale (Aziz et al. 2017). Numerous studies have been reported using microfluidics to mimic the human physiological systems in small chips, where they are referred to as microphysiological systems or organ-on-chip (Aziz et al. 2017). In these systems, it is possible to design microenvironments composed of different types of cells and promote their interaction as well as to mimic the interfaces between tissue-engineered constructs and physicochemical processes (Zheng et al. 2016).

Moreover, one of the main advantages of organ-onchip is the real-time monitoring of cell culture conditions $\left(\mathrm{CO}_{2}\right.$ rate, temperature, infusion and withdrawal of medium, injection of drugs, and nanoparticles) (Zhang et al. 2018). Furthermore, organ-on-chips are platforms that promote high-throughput analysis and big data collection, decrease response time, and are able to scale up (Novak et al. 2018). Moreover, several chambers with different types of cells can be designed and connected on the same platform (Oleaga et al. 2016). Named as body-on-chip, these platforms act as multi-tissue representative systems, providing important clues in drug assessment, cancer research, and nanotoxicology (Skardal et al. 2016). The main advantage is one be able to real-time monitoring, for instance, drug effects in secondary tissues predicting possible adverse effects (Oleaga et al. 2016).

It is known that COVID-19 infection can lead to cytokine storms (Jose and Manuel 2020). Thereby, body-on-chip devices are promising tools able to unravel and predict cytokine roles and other side effects of COVID-19 in several organs. Tay and coworkers (2016) extensively reviewed innovative advances in microfluidics to combat infectious diseases, including malaria, AIDS/HIV, tuberculosis, 


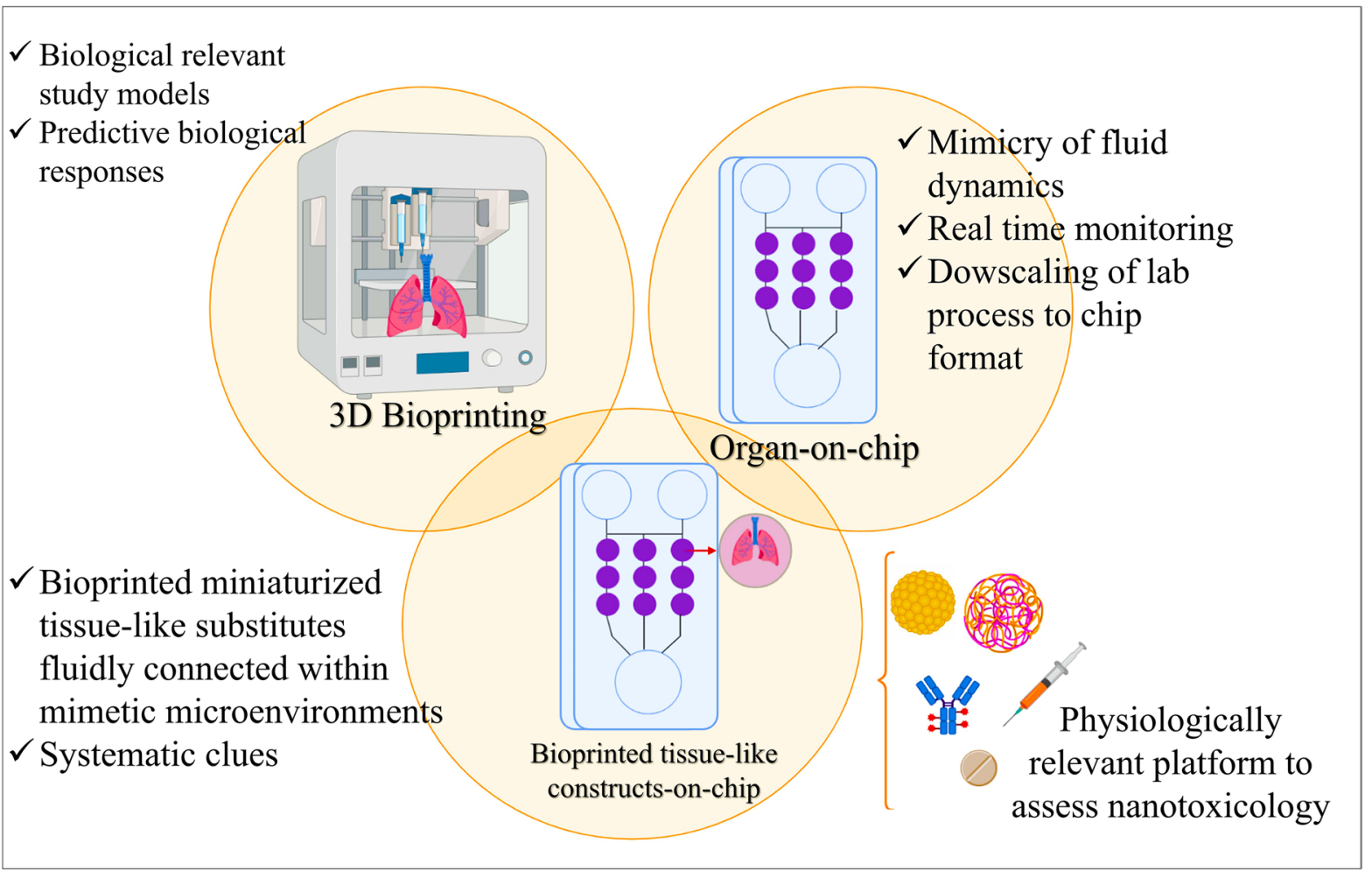

Fig. 4 Forefront technologies to assess nanosafety. 3D bioprinting promotes the biofabrication of structurally functional biological substitutes. Microphysiological systems or organon-chip devices mimic the microenvironment required for tis-

and dengue (Tay et al. 2016). Moreover, it is known that during COVID-19 disease, infiltration of immune and inflammatory cells (lymphocytes and monocytes) occurs in the lungs, as well as ultrastructural changes in bronchial mucosal epithelia and type II alveolar epithelia (Tian et al. 2020). Thus, using organ-on-chip devices, one is able to study the migration of these cells in real-time as well as to analyze the changes in the histoarchitecture. Other events were also seen in COVID-19 patients such as acute myocyte necrosis, SARS-CoV-2 neurotropism, and hepatic cell degeneration which could also be effectively assessed by using organ-on-chip technology.

The convergence between microfluidics and 3D bioprinting is already a reality (Ma et al. 2018b; Bhise et al. 2016). The synergism provides bioprinted miniaturized functional tissue-like substitutes fluidly connected within mimetic microenvironments where one is able to test several compounds, such as nano-designed drugs (Cong et al. 2020) or sue-engineered constructs to mature and respond to the input of several compounds. The convergence between booth technologies promotes a physiologically robust platform to assess and predict nanosafety

other compounds of choice, obtaining a systematic response, as shown in Fig. 4 (Bhise et al. 2016; Homan et al. 2016).

The in vitro preclinical phase determines whether the nanostructured polymer will continue to the next steps of the drug pipeline. Hence, the development of physiological relevant platforms to model the dynamism of the human body is mandatory. Bioprinting and microfluidics technologies have allowed researchers worldwide to achieve/understand biological events that were not possible with traditional cell culture methods, which make them excellent platforms to assess not only nanosafety but also the efficiency of the nanotherapeutic agents.

\section{Conclusion}

Nanotechnology can be of fundamental importance to combat life-threatening conditions. Considering the 
coronavirus disease (COVID-19) scenario and future outbreaks, nanotechnology can exert a pivotal role in several areas that include disinfection protocols, hospital clothing, and healthcare settings. One year after the COVID-19 outbreak, we only have a few vaccines that have been approved for coronavirus disease. Therefore, the development and design of new prophylactic and therapeutic drugs are mandatory.

The versatility of polymeric-based nanoparticle engineering can provide specificity tunable release kinetics and multimodal drug composition, allowing to overcome the common limitations encountered in traditional drug development. Nonetheless, steps such as regulation, safety, and the manufacture of the process should be optimized to achieve practical clinical applications.

Acknowledgements The images were created with BioRender.com.

Funding This work was supported by the Foundation for Research Support of the State of Rio de Janeiro (FAPERJ, Brazil), the Coordination for the Improvement of Higher Education Personnel (CAPES, Brazil), and the Brazilian National Council for Scientific and Technological Development (CNPq, Brazil).

Data availability Not applicable.

Code availability Not applicable.

\section{Declarations}

Conflict of interest The authors declare no competing interests.

\section{References}

Abo-zeid Y, Williams GR, Touabi L, McLean GR (2020) An investigation of rhinovirus infection on cellular uptake of poly (glycerol-adipate) nanoparticles. Int J Pharm. 589:119826

Aleissa MM, Silverman EA, Paredes Acosta LM, Nutt CT, Richterman A, Marty FM (2021) New perspectives on antimicrobial agents: remdesivir treatment for COVID-19. Antimicrob Agents Chemother 65

Al-Halifa S, Gauthier L, Arpin D, Bourgault S, Archambault D (2019) Nanoparticle-based vaccines against respiratory viruses. Front Immunol 10:1-11

Anselmo AC, Mitragotri S (2019) Nanoparticles in the clinic: an update. Bioeng Transl Med 4

Attia MF, Anton N, Wallyn J, Omran Z, Vandamme TF (2019) An overview of active and passive targeting strategies to improve the nanocarriers efficiency to tumour sites. J Pharm Pharmacol 71:1185-1198
Aziz A, Geng C, Fu M, Yu X, Qin K, Liu B (2017) The role of microfluidics for organ on chip simulations. Bioengineering 4:39

Bandopadhyay S, Manchanda S, Chandra A, Ali J, Deb PK (2019) Overview of different carrier systems for advanced drug delivery. Elsevier Inc.

Bhardwaj P, Bhatia E, Sharma S, Ahamad N, Banerjee R (2020) Advancements in prophylactic and therapeutic nanovaccines. Acta Biomater 108:1-21

Bhise NS, Manoharan V, Massa S, Tamayol A, Ghaderi M, Miscuglio M et al (2016) A liver-on-a-chip platform with bioprinted hepatic spheroids. Biofabrication. 8:014101

Chan LJ, Feeney OM, Leong NJ, McLeod VM, Porter CJH, Williams CC et al (2017) An evaluation of optimal PEGylation strategies for maximizing the lymphatic exposure and antiviral activity of interferon after subcutaneous administration. Biomacromol 18:2866-2875

Chauhan G, Madou MJ, Kalra S, Chopra V, Ghosh D, Martinez-Chapa SO (2020) Nanotechnology for COVID19: therapeutics and vaccine research. ACS Nano 14:7760-7782

Cong Y, Han X, Wang Y, Chen Z, Lu Y, Liu T et al (2020) Drug toxicity evaluation based on organ-on-a-chip technology: a review. Micromachines 11:381

Da Silva AL, Santos RS, Xisto DG, Alonso SDV, Morales MM, Rocco PRM (2013) Nanoparticle-based therapy for respiratory diseases. An Acad Bras Cienc 85:137-146

Di Gennaro F, Pizzol D, Marotta C, Antunes M, Racalbuto V, Veronese $\mathrm{N}$ et al (2020) Coronavirus diseases (COVID19) current status and future perspectives: a narrative review. Int J Environ Res Public Health 17:2690

Drobysh M, Ramanaviciene A, Viter R, Ramanavicius A (2021) Affinity sensors for the diagnosis of covid-19. Micromachines 12:1-19

Eslami G, Mousaviasl S, Radmanesh E, Jelvay S, Bitaraf S, Simmons B et al (2020) The impact of sofosbuvir/ daclatasvir or ribavirin in patients with severe COVID-19. J Antimicrob Chemother 75:3366-3372

FDA approves first treatment for COVID-19. FDA Website 2020. https://www.fda.gov/news-events/press-annou ncements/fda-approves-first-treatment-covid-19 (accessed April 13, 2021).

Fontanet A, Autran B, Lina B, Kieny MP, Karim SSA, Sridhar D (2021) SARS-CoV-2 variants and ending the COVID19 pandemic. Lancet 397:952-954

Forni G, Mantovani A (2021) COVID-19 vaccines: where we stand and challenges ahead. Cell Death Differ 28:626-639

Gao D, Guo X, Zhang X, Chen S, Wang Y, Chen T, et al (2020) Multifunctional phototheranostic nanomedicine for cancer imaging and treatment. vol. 5. Elsevier Ltd

Gorle AP, Gattani SG (2009) Design and evaluation of polymeric ocular drug delivery system. Chem Pharm Bull (tokyo) 57:914-919

Gref R, Minamitake Y, Peracchia M, Trubetskoy V, Torchilin V, Langer R (1994) Biodegradable long-circulating polymeric nanospheres. Science 263:1600-1603

Grigoryan B, Paulsen SJ, Corbett DC, Sazer DW, Fortin CL, Zaita AJ et al (2019) Multivascular networks and functional intravascular topologies within biocompatible hydrogels. Science 364:458-464 
Homan KA, Kolesky DB, Skylar-Scott MA, Herrmann J, Obuobi H, Moisan A et al (2016) Bioprinting of 3D convoluted renal proximal tubules on perfusable chips. Sci Rep 6:34845

Islam MR, Hoque MN, Rahman MS, Alam ASMRU, Akther M, Puspo JA et al (2020) Genome-wide analysis of SARS-CoV-2 virus strains circulating worldwide implicates heterogeneity. Sci Rep 10:14004

Jagaran K, Singh M (2020) Nanomedicine for covid-19: potential of copper nanoparticles. Biointerface Res Appl Chem 11:10716-10728

Jain A, Kumari R, Tiwari A, Verma A, Tripathi A, Shrivastava A et al (2018) Nanocarrier based advances in drug delivery to tumor: an overview. Curr Drug Targets 19:1498-1518

Jazayeri MH, Amani H, Pourfatollah AA, Pazoki-Toroudi H, Sedighimoghaddam B (2016) Various methods of gold nanoparticles (GNPs) conjugation to antibodies. Sens Bio-Sensing Res 9:17-22

Jose RJ, Manuel A (2020) COVID-19 cytokine storm: the interplay between inflammation and coagulation. Lancet Respir Med 8:e46-e47

Kalyane D, Maheshwari R, Raval N, Chauhan AS, Tekade RK (2018) Transportation and biointeraction properties in nanomaterials across biological systems. Elsevier Inc.

Khoris IM, Ganganboina AB, Suzuki T, Park EY (2021) Selfassembled chromogen-loaded polymeric cocoon for respiratory virus detection. Nanoscale 13:388-396

Kim MG, Park JY, Shon Y, Kim G, Shim G, Oh YK (2014) Nanotechnology and vaccine development. Asian J Pharm Sci 9:227-235

Kulterer MR, Reischl M, Reichel VE, Hribernik S, Wu M, Köstler $S$ et al (2011) Nanoprecipitation of cellulose acetate using solvent/nonsolvent mixtures as dispersive media. Colloids Surfaces A Physicochem Eng Asp 375:23-29

Kumari A, Yadav SK, Yadav SC (2010) Biodegradable polymeric nanoparticles based drug delivery systems. Colloids Surfaces B Biointerfaces 75:1-18

Li B, Wang W, Song W, Zhao Z, Tan Q, Zhao Z et al (2021) Antiviral and anti-inflammatory treatment with multifunctional alveolar macrophage-like nanoparticles in a surrogate mouse model of COVID-19. Adv Sci 2003556:1-14

Ma J, Wang Y, Liu J (2018a) Bioprinting of 3D tissues/organs combined with microfluidics. RSC Adv 8:21712-21727

Ma X, Liu J, Zhu W, Tang M, Lawrence N, Yu C et al (2018b) 3D bioprinting of functional tissue models for personalized drug screening and in vitro disease modeling. Adv Drug Deliv Rev 132:235-251

Manchanda S, Das N, Chandra A, Bandyopadhyay S, Chaurasia S (2019) Fabrication of advanced parenteral drugdelivery systems. Elsevier Inc.; 2019

Manek E, Petroianu GA (2020) Brain delivery of antidotes by polymeric nanoparticles. J Appl Toxicol 1-13

Matsuo Y, Ishihara T, Ishizaki J, Miyamoto K, Higaki M, Yamashita N (2009) Effect of betamethasone phosphate loaded polymeric nanoparticles on a murine asthma model. Cell Immunol 260:33-38

Medhi R, Srinoi P, Ngo N, Tran HV, Lee TR (2020) Nanoparticle-based strategies to combat COVID-19. ACS Appl Nano Mater 3:8557-8580
Milane L, Amiji M (2021) Clinical approval of nanotechnology-based SARS-CoV-2 mRNA vaccines: impact on translational nanomedicine. Drug Deliv Transl Res

Militky J, Novak O, Kremenakova D, Wiener J, Venkataraman M, Zhu G et al (2021) A review of impact of textile research on protective face masks. Materials (basel) 14:1-16

Montanheiro TL do A, Ribas RG, Montagna LS, de Menezes BRC, Schatkoski VM, Rodrigues KF et al (2020) A brief review concerning the latest advances in the influence of nanoparticle reinforcement into polymeric-matrix biomaterials. J Biomater Sci Polym Ed. 31:1869-93

Mousavizadeh L, Ghasemi S (2020) Genotype and phenotype of COVID-19: their roles in pathogenesis. J Microbiol Immunol Infect

Nanomedicine and the COVID-19 vaccines. Nat Nanotechnol. 2020;15:963.

Neufurth M, Wang X, Tolba E, Lieberwirth I, Wang S, Schröder HC et al (2020) The inorganic polymer, polyphosphate, blocks binding of SARS-CoV-2 spike protein to ACE2 receptor at physiological concentrations. Biochem Pharmacol. 182:114215

Neufurth M, Wang X, Wang S, Schröder HC, Müller WEG (2021) Caged dexamethasone/quercetin nanoparticles, formed of the morphogenetic active inorganic polyphosphate, are strong inducers of MUC5AC. Mar Drugs 19:64

Novak R, Didier M, Calamari E, Ng CF, Choe Y, Clauson SL, et al (2018) Scalable fabrication of stretchable, dual channel, microfluidic organ chips. J Vis Exp

Oleaga C, Bernabini C, Smith AST, Srinivasan B, Jackson M, McLamb W et al (2016) Multi-organ toxicity demonstration in a functional human in vitro system composed of four organs. Sci Rep 6:20030

Organization WH. WHO coronavirus (COVID-19) dashboard n.d. https://covid19.who.int/ (accessed May 10, 2021).

Park JY, Jang J, Kang H-W (2018) 3D Bioprinting and its application to organ-on-a-chip. Microelectron Eng 200:1-11

Parvez MK, Parveen S (2017) Evolution and emergence of pathogenic viruses: past, present, and future. Intervirology 60:1-7

Patra JK, Das G, Fraceto LF, Campos EVR, Rodriguez-Torres M del P, Acosta-Torres LS et al (2018) Nano based drug delivery systems: recent developments and future prospects. J Nanobiotechnology. 16:71

Peng W, Datta P, Ayan B, Ozbolat V, Sosnoski D, Ozbolat IT (2017) 3D bioprinting for drug discovery and development in pharmaceutics. Acta Biomater 57:26-46

Pinto MCC, Cipolatti EP, Manoel EA, Freire DMG, Becer ÇR, Pinto JC (2020) Production of new functionalized polymer nanoparticles and use for manufacture of novel nanobiocatalysts. Macromol Mater Eng 305:2000065

Pooladanda V, Thatikonda S, Godugu C (2020) The current understanding and potential therapeutic options to combat COVID-19. Life Sci. 254:117765

Rahmani H, Davoudi-Monfared E, Nourian A, Nabiee M, Sadeghi S, Khalili H et al (2020) Comparing outcomes of hospitalized patients with moderate and severe COVID-19 following treatment with hydroxychloroquine plus atazanavir/ritonavir. DARU, J Pharm Sci 28:625-634 
Rana MM (2021) Polymer-based nano-therapies to combat COVID-19 related respiratory injury: progress, prospects, and challenges. J Biomater Sci Polym Ed 32:1219-1249

Rezvantalab S, Drude NI, Moraveji MK, Güvener N, Koons EK, Shi Y et al (2018) PLGA-based nanoparticles in cancer treatment. Front Pharmacol 9:1-19

Ruiz-Hitzky E, Darder M, Wicklein B, Ruiz-Garcia C, MartínSampedro R, del Real G et al (2020) Nanotechnology Responses to COVID-19. Adv Healthc Mater 9:1-26

Sahandi Zangabad P, Karimi M, Mehdizadeh F, Malekzad H, Ghasemi A, Bahrami S et al (2017) Nanocaged platforms: modification, drug delivery and nanotoxicity. Opening synthetic cages to release the tiger. Nanoscale. 9:1356-92

Saraogi GK, Gupta P, Gupta UD, Jain NK, Agrawal GP (2010) Gelatin nanocarriers as potential vectors for effective management of tuberculosis. Int J Pharm 385:143-149

Siewert CD, Haas H, Cornet V, Nogueira SS, Nawroth T, Uebbing $\mathrm{L}$ et al (2020) Hybrid biopolymer and lipid nanoparticles with improved transfection efficacy for mRNA. Cells 9:1-19

Singh AK, Mishra G, Maurya A, Kulkarni GT, Awasthi R (2020) Biofabrication: an interesting tool to create in vitro model for COVID-19 drug targets. Med Hypotheses. 144:110059

Skardal A, Devarasetty M, Forsythe S, Atala A, Soker S (2016) A reductionist metastasis-on-a-chip platform for in vitro tumor progression modeling and drug screening. Biotechnol Bioeng 113:2020-2032

Sun R, Luo Q, Wang Y, Chu H, Li X, Shen Z et al (2021) Supramolecular engineering of polymeric nanodrugs for antitumor chemotherapy. Chem Eng J. 416:127968

Sur S, Rathore A, Dave V, Reddy KR, Chouhan RS, Sadhu V (2019) Recent developments in functionalized polymer nanoparticles for efficient drug delivery system. NanoStructures \& Nano-Objects 20:100397

Tay A, Pavesi A, Yazdi SR, Lim CT, Warkiani ME (2016) Advances in microfluidics in combating infectious diseases. Biotechnol Adv 34:404-421

Tian S, Xiong Y, Liu H, Niu L, Guo J, Liao M et al (2020) Pathological study of the 2019 novel coronavirus disease (COVID-19) through postmortem core biopsies. Mod Pathol 33:1007-1014

Trampe E, Koren K, Akkineni AR, Senwitz C, Krujatz F, Lode A et al (2018) Functionalized bioink with optical sensor nanoparticles for $\mathrm{O} 2$ imaging in 3D-bioprinted constructs. Adv Funct Mater 28:1804411

Udugama B, Kadhiresan P, Kozlowski HN, Malekjahani A, Osborne M, Li VYC et al (2020) Diagnosing COVID19: the disease and tools for detection. ACS Nano 14:3822-3835
Veronese FM, Mero A (2008) The impact of PEGylation on biological therapies. BioDrugs 22:315-329

Wang G, Yang L, Li C, Yu H, He Z, Yang C et al (2021) Novel strategy to prepare fluorescent polymeric nanoparticles based on aggregation-induced emission: via precipitation polymerization for fluorescent lateral flow assay. Mater Chem Front 5:2452-2458

Wang H, Zhou Y, Sun Q, Zhou C, Hu S, Lenahan C, et al (2021) Update on nanoparticle-based drug delivery system for anti-inflammatory treatment. Front Bioeng Biotechnol 9

Weiss C, Carriere M, Fusco L, Capua I, Regla-Nava JA, Pasquali $M$ et al (2020) Toward nanotechnology-enabled approaches against the COVID-19 pandemic. ACS Nano 14:6383-6406

Witika BA, Makoni PA, Mweetwa LL, Ntemi PV, Chikukwa MTR, Matafwali SK et al (2020) Nano-biomimetic drug delivery vehicles: potential approaches for COVID-19 treatment. Molecules 25:1-20

Wu J, Wang H, Li B (2020) Structure-aided ACEI-capped remdesivir-loaded novel PLGA nanoparticles: toward a computational simulation design for anti-SARS-CoV-2 therapy. Phys Chem Chem Phys 22:28434-28439

Yang D (2021) Application of nanotechnology in the COVID19 pandemic. Int J Nanomedicine 16:623-649

Yetisgin AA, Cetinel S, Zuvin M, Kosar A, Kutlu O (2020) Therapeutic nanoparticles and their targeted delivery applications. Molecules 25:2193

Zhang M, Hong Y, Chen W, Wang C (2017) Polymers for DNA vaccine delivery. ACS Biomater Sci Eng 3:108-125

Zhang B, Korolj A, Lai BFL, Radisic M (2018) Advances in organ-on-a-chip engineering. Nat Rev Mater 3:257-278

Zhang Q, Honko A, Zhou J, Gong H, Downs SN, Vasquez JH et al (2020) Cellular nanosponges inhibit SARS-CoV-2 infectivity. Nano Lett 20:5570-5574

Zheng F, Fu F, Cheng Y, Wang C, Zhao Y, Gu Z (2016) Organon-a-chip systems: microengineering to biomimic living systems. Small 12:2253-2282

Zhou J, Kroll AV, Holay M, Fang RH, Zhang L (2020) Biomimetic nanotechnology toward personalized vaccines. Adv Mater 32:1901255

Publisher's note Springer Nature remains neutral with regard to jurisdictional claims in published maps and institutional affiliations. 